\title{
Energy Releases from Accreting Superdense Compact Objects in AGNs
}

\author{
Avetis Abel Sadoyan \\ Yerevan State University, Alex Manoogian 1, Yerevan 375025, Armenia, \\ Email:asadoyan@www.physdep.r.am
}

\begin{abstract}
We consider an accreting central symmetric object that is near to the Eddington limit, i.e., the luminosity pressure is balancing the gravity. In special cases, a layer of accreting matter will be formed near the surface of a centrally symmetric object. We discuss the conditions of formation of the layer, its inner structure and stability. If the luminosity pressure will be reduced for some reason, then the layer will collapse immediately, releasing an enormous quantity (up to $10^{42} \mathrm{erg} / \mathrm{sec}$ ) of energy. We discuss some possible scenarios for energy releases.
\end{abstract}

\section{Introduction}

We are considering spherically symmetric accretion on superdense configurations that are near the Eddington Limit. Let's take Shchwarzshild coordinates with

$$
\mathrm{g}_{00}=1-\frac{r_{g}}{r} ; \mathrm{g}_{11}=-\frac{1}{1-\frac{r g}{r}} ; \mathrm{g}_{22}=-r^{2} ; \mathrm{g}_{33}=-r^{2} \sin ^{2} \theta ;
$$

where $r_{g}=\frac{2 G M}{c^{2}}, M$ is the mass of the central body, and $\mathrm{c}$ is the speed of light. The pressure of radiation has no evident impact on the parameters of the superdense configuration, except the thin layer of the Ae-shell near the surface. One can easily derive the equation for stationary accreting matter with radiation pressure as

$$
\frac{d P}{d r}=-\frac{G M \rho \delta}{r^{2}\left(1-\frac{r_{g}}{r}\right)}
$$

where $\rho$ is the density of infalling matter, $\delta=1-\frac{L}{L_{e}}$, and $\mathrm{L}$ is the flow of radiated energy at a distance $r$ from the center in units of time.

The definition for the Eddington Limit $\mathrm{L}_{e}$ in the fully relativistic case is

$L_{e}(r)=\frac{4 \pi c G M}{\chi \sqrt{1-\frac{r g}{r}}}$

where $\chi$ is the optical opacity of infalling matter, while in Newtonian Gravitation $L_{e}$ is constant and equals $L_{E N}=\frac{4 \pi G M c}{\chi}$;

As one can see in the region where $\delta$ is positive we have accretion; where negative, the resulting forces break down the accretion and could stop the infalling matter and even throw it back. The evolution can go by different scenarios depending on the differential accretion, the kind of the infalling matter, and the initial velocity at infinity. 


\section{Possible Scenarios for Bursts}

There are several scenarios for the evolution of a central configuration that is near the Eddington Limit; let's discuss four interesting cases:

\section{Scenario 1 ("Greenhouse Effect")}

Energy release is conditioned by the thermal state of the central configuration, that is an enormous container of thermal energy. The accreting matter stops, forming a "hanging layer" that closes the way out for radiation. The hadron nuclei heat up, a burst takes place, nuclei lose their thermal energy through the work carried out during the outburst of the mass and a significantly small part through emission. The burst stops and the accretion to the surface starts just at the very moment when the luminosity becomes smaller than the Eddington Limit. After a while (quiet period of the burster life) the flow of energy to the hadron nuclei decreases and when the luminosity reaches the Eddington Limit a new burst occurs.

This scenario could be repeated several times and does not need a permanent source of accreting matter, i.e., a companion to feed the acretion, because falling matter plays the role of simple regulator for the radiation.

\section{Scenario 2 ("Falling Matter")}

Energy release is conditioned by the temp of accreting matter. The accretion rate decreases due to the gap, the luminosity becomes less than the Eddington limit and the hanging matter collapses onto the surface of the central engine, releasing all the gravitational energy for the accumulated mass. The luminosity increases and stops the further infall of matter.

\section{Scenario 3 (X-Ray Bursters)}

This a hybrid of the first and second scenarios. When the accumulated accreting matter falls down it heats up the central configuration, which outbursts, throwing back also the thin layer of Ae-shell and opening the hot core (G. Sahakyan, G. Alojants, A. Sarkissian 1991). We record an X-ray burst.

\section{Scenario 4 (Soft Gamma Repeaters)}

If in Scenario 3 the energy of the outburst is high enough to accelerate the burst matter to velocites such that a shock wave will occur, then we shall have a soft gamma-ray burst. The shock wave will pass through the accreting matter and will produce non-thermal gamma emission (Sadoyan \& Alodjanc 2000).

As the opacity for electrons is much higher than that for protons, this is a good solution for the "Baryon Load Problem" in GRBs. Computer simulations of the model make it possible to understand the origin of intense trains of recurrent bursts, when the intervals between the bursts decrease at times to such an extent as to become comparable to the duration of the bursts themselves.

\section{References}

Sahakyan, G., Alojants, G., Sarkissian,A., 1991, Astrofizika, v.34,21-40

Sadoyan, A.A., Alodjanc G.A., 2000, in Texas 20 Conf. Proc.,112 